\title{
A Case of COVID-19 Mortality in a Pregnant Woman with Diabetes Ketoacidosis
}

\author{
Soheila Aminimoghaddam ${ }^{1}$, Setareh Nasiri ${ }^{1}$, Aida Abrari ${ }^{1}$, Maryam Yazdizadeh ${ }^{1} * \mathbb{D}$, Romina Rashidi $^{2}$ \\ Received: 12 Mar 2021 \\ Published: 21 Oct 2021
}

\begin{abstract}
SARS-CoV-2 is a newly identified virus that causes COVID 19, spreading very fast in the world. Uncontrolled diabetes in pregnancy can increase the risk of pregnancy outcomes. Pregnant women are at high risk of developing a viral infection, like SARSCOV and on the other hand, diabetes ketoacidosis (DKA) which is coupled with COVID-19, can increase maternal mortality. The patient was a 27-years-old female G3P1L1Ab1 with a history of a previous cesarean section. On 26 March 2020, a fetal ultrasound revealed intra-uterine fetal death (IUFD) and also diagnosis of DKA and COVID-19 in the patient that she was expired eventually due to the uncontrolled DKA. In this case report, a pregnant woman with a diagnosis of IUFD, DKA and COVID-19 simultaneously is described. To the best of the authors of this paper's knowledge, no previous work has been reported for the comorbidity of diabetes and COVID-19 in pregnancy, but it seems that the coincidence of the above-mentioned diseases can delay the recovery period and also can increases maternal and fetal mortality. When DKA and COVID-19 appear in the patient simultaneously, we cannot control DKA by the routine protocol treatments of DKA which were used formerly.
\end{abstract}

Keywords: COVID-19, IUFD, Diabetes ketoacidosis, Pregnancy.

Conflicts of Interest: None declared

Funding: Dr Maryam Yazdizadeh.

*This work has been published under CC BY-NC-SA 1.0 license.

Copyright $\odot$ Iran University of Medical Sciences

Cite this article as: Aminimoghaddam S, , Nasiri S, Abrari A, Yazdizadeh M, Rashidi R. A Case of COVID-19 Mortality in a Pregnant Woman with Diabetes Ketoacidosis. Med J Islam Repub Iran. 2021 (21 Oct);35:139. https://doi.org/10.47176/mjiri.35.139

\section{Introduction}

The coronavirus infectious disease in 2019 (COVID19 ), which is caused by the novel coronavirus (severe acute respiratory syndrome coronavirus 2 (SARS-CoV2)) has been increasing continuously with more than a million confirmed cases and more than 45 thousand deaths globally. Since the high prevalence of diabetes is increasing, the different aspects of this subject became more important, as in most parts of the world, there exist restrictions on patient mobility in order to control the pandemic (1). Accordin $g$ to the evidence, COVID-19 has been increasing due to the person-to-person transmission

Corresponding author: Dr Maryam Yazdizadeh, Yazdizadeh.m@iums.ac.ir

1. Department of Obstetrics and Gynecology, Faculty of Medicine, Iran University of Medical Sciences, Tehran, Iran

2. Department of Obstetrics and Gynecology, Faculty of Medicine, Gilan University of Medical Sciences, Gilan, Iran in hospitals and family settings. On the other hand, the transmission systems are not completely defined since there are no effective treatments or vaccines yet (2). Diabetes during pregnancy shows health threats for both the mother and fetus and can be cllassified into three types of diabetes which are type 1 diabetes, type 2 diabetes, and gestational diabetes. Uncontrolled diabetes in pregnancy can increase the risk of undesirable pregnancy outcomes including macrosomia, neonatal hypoglycemia, congenital anomaly, and prenatal mortality (3). In the previous studies, pregnant women are at a high risk of the progressive.

$\uparrow$ What is "already known" in this topic:

Previous reports have shown good pregnancy prognosis for covid-19 infected pregnant wemen with negative past medical history of diabetes.

\section{$\rightarrow$ What this article adds:}

Our findings suggest that COVID-19 infection can convert diabetes to DKA and the DKA cannot be controlled by the routine protocol treatment of DKA and also Uncontrolled diabetes in pregnancy can increase the risk of undesirable pregnancy outcomes. 
viral infection, like influenza-A, H1N1, SARS-CoV, Middle East Respiratory Syndrome coronavirus (MERS$\mathrm{CoV}$ ), and Ebola virus, and it also appears to have worse clinical outcomes like maternal mortality, stillbirth, spontaneous abortion, and preterm delivery, in comparison with the non-pregnant women. However, there exists only limited data on the clinical features for COVID-19 in pregnant women (4). There are evidence that show the incidence and severity of COVID-19 are increased in cases with diabetes and laboratory data also indicated that the patients of COVID-19 with diabetes are mostly associated with severe or critical disease varying from $14 \%$ to $32 \%$ in different studies (5-8).

COVID-19 can have an effect on the pathophysiology of diabetes; ACE-2 receptors are expressed in pancreatic islets and also infection with SARS CoV-1 has been seen to cause hyperglycemia in cases without pre-existing diabetes. Hyperglycemia was seen to persist for 3 years after the recovery period of SARS which is indicating transient damage to beta cells (9) and underlying diseases like diabetes coupled with COVID-19 significantly increases the risk of death (10). However, there are only limited data about the clinical features of COVID-19 in pregnant women (11). Due to the fact that the prevalence of COVID-19 in pregnancy increases, COVID-19 infection can convert diabetes to DKA and the DKA cannot be controlled by the routine protocol treatment of DKA. Therefore it finally causes death. Hence, monitoring of blood glucose level in the acute stage and also during the follow-up period of the patient by COVID-19 is important and the diabetes cases which are infected by COVID-19 are needed more attention in order to prevent the increase of BS and DKA in pregnancy.4

\section{Case Report}

On 26 March 2020, a 27-year-old female G3P1L1Ab1 with a history of a previous cesarean section was presented to Lolagar health center in Tehran city of Iran. By measuring the fundal height, it is revealed that the patient was 28 weeks pregnant. The reason for referring was the lack of proper management of the DKA and suspicion of COVID-19. Other symptoms were dyspnea, nausea and sporadic dry coughs from about ten days ago, and also complaints of reducing the fetal movement from two days

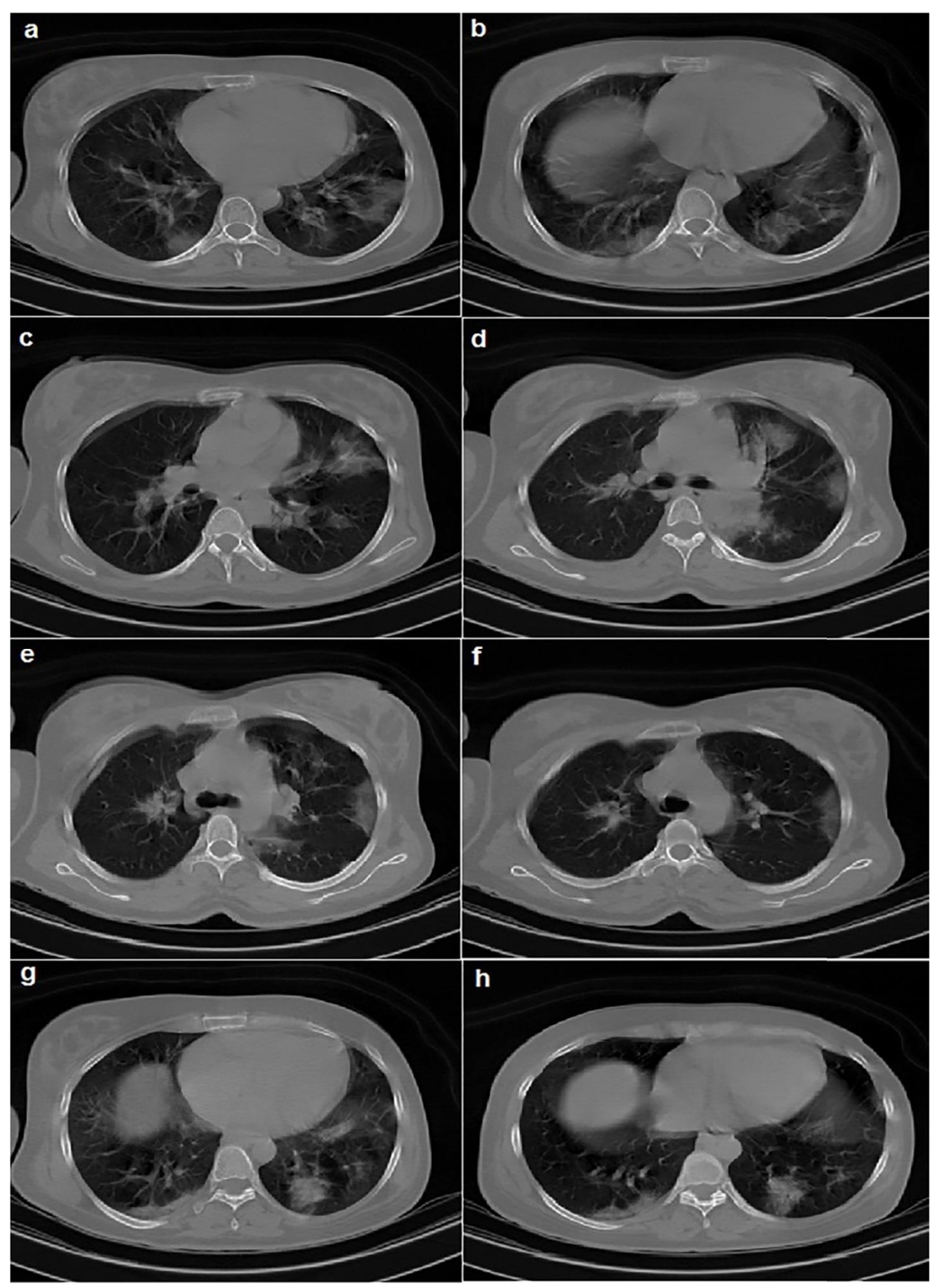

Fig. 1. CT scan on first day of admission which shows the bilateral, peripheral and patchy ground-glassopacities due to the evidence of COVID-19 
ago. The patient had insulin-dependent diabetes mellitus from 4 years ago and she did not use Lantus and regular insulin orderly and a suspected history of addiction was also perceived at the beginning of her presentation in Firoozgar hospital as well. She lived with her husband and there were no kindred between them before the marriage. She did not smoke or drink alcohol and she was illiterate. Physical examination showed a body temperature of $38.3^{\circ} \mathrm{C}$, a blood pressure of $135 / 84 \mathrm{~mm} \mathrm{Hg}$, a pulse rate of 108 beats per minute, respiratory rate of 22 breaths per minute, and oxygen saturation of $92 \%$ with a facial mask at $5 \mathrm{~L}$ per minute of oxygen. Fetal ultrasound revealed the IUFD and breech presentation with about 26 weeks and 4 days of gestational age by observing the $\mathrm{BS}=405 \mathrm{mg} / \mathrm{dl}, \mathrm{HCO}_{3}=3.5 \mathrm{~m} \mathrm{moll} / \mathrm{l}$, and $\mathrm{PH}=6.85$. With a diagnosis of DKA, she was transferred to the intensive care unit (ICU), and afterward, she was treated with regular insulin at the beginning (10 unit stat) and then 6 units per hour. The regular insulin was changed based on the electrolytes, BS level, rule out of COVID-19, and the other aspects of managing the DKA. Chest computed tomography (CT) scan demonstrated bilateral, peripheral and patchy ground-glass opacities on her first day of admission which elucidate the evidence of COVID-19 (Fig. 1). Therefore, she was isolated and 2 swab samples were collected from her throat and nasopharynx and the test was positive for SARS-CoV-2, whereas the blood culture and the urine culture analysis were negative. We also checked K q $12 \mathrm{~h}$ and BS q $6 \mathrm{~h}$. The other laboratory data are listed in Table 1.

The DKA protocol which is shown in Table 2 and COVID-19 treatment were initiated for the patient. The day after hospitalization, she underwent cesarean section due to the blood pressure of 140/95 and IUFD by suspicious of preeclampsia and a fettal breech presentation with a history of a previous cesarean section. Her dyspnea and O2 saturation level were getting worse day by day, despite she was intubated. Treatment with meropenem, vancomycin, ribavirin, kaletra, infliximab, hydroxyl chloroquine sulfate, hydrocortisone, methylprednisolone, Nacetylcysteine, and dextromethorphan (Table 2), and Lantus and regular insulin was started. The routine protocol treatments of DKA did not reduce her BS level because of the uncontrolled diabetes, COVID-19 and on the other hand, she did not use insulin before her admission. There exist both acute respiratory distress syndrome (ARDS) and end organ damage (according to increase of creatinine and liver function tests). Therefore, cardiac and pulmonary arrest occurred and the patient was expired despite of the standard cardiopulmonary resuscitation (CPR).

Table 1. Laboratory tests of the patient during the different days

\begin{tabular}{|c|c|c|c|c|c|}
\hline & $26 \mathrm{mar}$ & $28 \mathrm{mar}$ & $31 \mathrm{mar}$ & $3 a p r$ & 4apr \\
\hline WBC (per mm $\left.\mathrm{mm}^{3}\right)$ & $\begin{array}{c}9.100 \\
\text { gment }=84 \%\end{array}$ & $\begin{array}{c}8.500 \\
\text { Segment }=88.5 \%\end{array}$ & $\begin{array}{c}8.500 \\
\text { gment }=90.6 \%\end{array}$ & $\begin{array}{c}14.700 \\
\text { Segmentt }=91 \%\end{array}$ & $\begin{array}{c}24.500 \\
\text { Segment }=92.9 \%\end{array}$ \\
\hline $\mathrm{HB}(\mathrm{g} / \mathrm{dl})$ & 7.8 & 11.4 & 9.2 & 7.9 & 7.9 \\
\hline $\operatorname{PLT}\left(\right.$ per $\mathrm{mm}^{3}$ ) & 208000 & 138000 & 121000 & 620100 & 50000 \\
\hline LDH (u/liter) & 1156 & 1191 & 2430 & 6456 & 6750 \\
\hline AST (u/liter) & 56 & 32 & 33 & 832 & 3978 \\
\hline $\operatorname{ALT}(\mathrm{u} /$ liter $)$ & 14 & 15 & 28 & 219 & 594 \\
\hline $\mathrm{BS}(\mathrm{mg} / \mathrm{dl})$ & 66 , and 212 & 340 , and 164 & 303 , and 201 & 275 , and 248 & 308, 1nd 374 \\
\hline $\mathrm{PH}$ & 6.85 & 7.24 & 7.26 & 6.97 & 7.37 \\
\hline $\mathrm{HCO}_{3}(\mathrm{~m} \mathrm{moll} / \mathrm{l})$ & 3.5 & 8.3 & 16.6 & 1.9 & 19.8 \\
\hline Urine analysis & Ketone $=+1$ & Ketone $=$ negative & Ketone $=+1$ & Ketone $=+1$ & Ketone $=+1$ \\
\hline $\mathrm{K}(\mathrm{m} \mathrm{moll} / \mathrm{l})$ & 5.3 , and 4.9 & 3.2, and 4 & 3.8 , and 3.6 & 4.5, and 3.9 & 4.5, and 5.4 \\
\hline $\mathrm{Cr}(\mathrm{mg} / \mathrm{dl})$ & 0.9 & 0.6 & 0.5 & 1.3 & 1.4 \\
\hline PT (sec) & 13.8 & 14.5 & 13.5 & 36.9 & 39.9 \\
\hline PTT (sec) & 32 & 35 & 38 & 54 & 77 \\
\hline INR & 1.3 & 1.4 & 1.3 & 2.11 & 3.12 \\
\hline $\operatorname{Mg}(\mathrm{mg} / \mathrm{dl})$ & 2 & 1.8 & 2 & 1.8 & 1.7 \\
\hline
\end{tabular}

Table 2. Drugs that are prescribed for the patient during the hospitalization

\begin{tabular}{|c|c|}
\hline Drugs & Dose \\
\hline Amp meropenem & 1gr IV (q $8 \mathrm{~h})$ \\
\hline Amp vancomycin & 1 gr IV (q 12 h) \\
\hline Tab ribavirin & $600 \mathrm{mg} / \mathrm{po}(\mathrm{q} 12 \mathrm{~h})$ \\
\hline Tab kaletra (lopinavir / ritonavir) & 200/50mg/PO (q 12 h) \\
\hline Amp infliximab & $300 \mathrm{mg} / \mathrm{IV} / \mathrm{stat}$ \\
\hline Tab hydroxyl chloroquine sulfate & $800 \mathrm{mg} / \mathrm{po} / \mathrm{stat}$ \\
\hline Amp hydrocortisone & $100 \mathrm{mg} / \mathrm{IV}$ (q 12 h) \\
\hline Amp methylprednisolone & $500 \mathrm{MG} / \mathrm{IV} / \mathrm{stat}$ then $40 \mathrm{mg}(\mathrm{q} 12 \mathrm{~h}) / \mathrm{BD}$ \\
\hline Amp pantoprazole & $40 \mathrm{mg} / \mathrm{IV}$ (q12h) \\
\hline $\mathrm{Tab}$ rosuvastatin & $20 \mathrm{mg} / \mathrm{po} /$ daily \\
\hline Amp N-acetyl cysteine & $1 \mathrm{gr} / \mathrm{IV}(\mathrm{q} \mathbb{1 1} 2 \mathrm{~h})$ \\
\hline Amp apotel & $1 \mathrm{gr} / \mathrm{IV} /(\mathrm{q} 8 \mathrm{~h})$ If $\mathrm{T} \geq 38 \mathrm{C}^{\circ}$ \\
\hline Syr dextromethorphan & $5 \mathrm{cc} / \mathrm{po} /(\mathrm{q} 8 \mathrm{~h})$ \\
\hline Serum therapy $/ \mathrm{KCl} / \mathrm{mgso} 4$ & Base on electrolytes and BS \\
\hline Drip insulin regular & Base on electroly'tes and BS \\
\hline Insulin Lantus & 10u/SC/daily \\
\hline Amp heparin & $5000 \mathrm{u} / \mathrm{sc}(\mathrm{q} 12 \mathrm{~h})$ \\
\hline Tab acetylcysteine & $600 \mathrm{mg} /$ po (q $12 \mathrm{~h})$ \\
\hline
\end{tabular}




\section{Discussion}

that she underwent cesarean section due to the blood pressure of 140/95, IUFD by suspicious of preeclampsia, and a fetal breech presentation with a history of a previous cesarean section. The routine protocol treatment of COVID- 19 and ARDS was started, but the treatment did not reduce her BS level which is due to the concurrent suffering of COVID-19 and diabetes, the uncontrolled diabetes, and also not utilizing the regular insulin before her admission. Finally, she was expired with diagnosis of ARDS, end organ damages, and uncontrolled BS. To the best of the authors' knowledge, no previous work has been reported on the comorbidity of diabetes and COVID19 in pregnancy, but there were limited similar subjects in non-pregnant patients. In retrospective study by Akhtar et al. in 2019 (10), it is concluded that the maternal, fetal, and neonatal outcomes of patients who were infected in late pregnancy, appeared very good. The result of this study is different with our result and it was predictable due to the non DKA association. In another retrospective study by Nan et al. (12) in Wuhan in 7 pregnant women with COVID-19 having mean gestational age of 39 weeks and 1 day and also negative past medical history of diabetes, they reported that the maternal, fetal, and neonatal consequences of patients who were infected in late pregnancy were good which is different with our result and it was foreseeable for the sake of non DKA association. In a case report study, Iqbal et al. (13) in 2020, an uncomplicated vaginal delivery in a pregnant patient with 34 years old and 39 weeks of gestational age with covid-19 by negative past medical history of diabetes was reported in the United States. In retrospective cohort study, Fei et al. (14) in 2019 reported that the diabetes was the second comorbidity with COVID-19 that led to non-pregnant patient death. In our study it seems that COVID-19 infection can covert diabetes to DKA which cannot be controlled by the routine protocol treatment of DKA and it finally leads to death. In another retrospective study by Huijun Chen et al. (15), nine pregnant by COVID-19 were investigated in Zhongnan hospital in 2020 by the negative past medical history of diabetes. The disease like pneumonia and severe COVID-19 were not seen and none of the patients died. Nine livebirths were recorded. No neonatal asphyxia was observed in newborn babies. The results confirm the main hypothesis of our study which is comorbidity of diabetes and COVID-19 in pregnant and it can increase the risk of maternal mortality and IUFD. The result of study by Awadhesh et al. (16) in 2020 in India was similar to our result which is due to the evidence of increased incidence and severity of COVID-19 in non-pregnant patients by diabetes. It seems COVID-19 could have effect on the pathophysiology of diabetes. BS control is important not only for patients who are infected with COVID-19, but also for those without the disease. Xiaotong et al. (17) in 2020 in Suzhou reported a case of a 28 years old pregnant woman by 30 -week gestational age with the 2019 novel coronavirus (COVID-19) without the past medical history of diabetes, and cesarean section who was delivering a healthy infant. Due to the intra uterine fetal death (IUFD) in our case, the result of this study is different with our result which can confirm our hypothesis.

\section{Conclusion}

Our study in Iran suggests insight into the relevant clinical and laboratory findings of this novel entity. The studied case shows the importance of the diagnosis of coronavirus disease and tough protocols in establishing elimination of infection in order to simplify removal of isolation precautions and also trusty return of the patients to the society. The topic is relevant for controlling disease spread and enhancing public safety. It may be important to monitor the blood glucose level in acute stage and during the follow up period of the patient by COVID-19 as well. The diabetes cases which are infected by COVID-19 are needed more attention in order to prevent the rise of BS and DKA in pregnancy. Future research is urgently needed to provide a better understanding of the association between COVID-19, diabetes, DKA, and a new clinical management.

\section{Conflict of Interests}

The authors declare that they have no competing interests.

\section{References}

1. Chan J, Yuan S, Kok K, Wang K, Chu H, Yang J, et al. A familial cluster of pneumonia associated with the 2019 novel coronavirus indicating person-to-person transmission: a study of a family cluster. Lancet. 2020;395:514-523.

2. Li Q, Guan X, Wu P, Wang X, Zhou L. Early transmission dynamics in Wuhan, China, of novel coronavirus-infected pneumonia. N Engl J Med. 2020;382:1199-207.

3. Torpy J, Lynm C, Glass R. Glass Pregnancy and diabetes JAMA. 2008;299(21): 2590-2590.

4. Olgun NS. Viral infections in pregnancy: a focus on Ebola virus. Curr Pharm Des. 2018;24:993-8.

5. CDC COVID-19 Response Teann. Preliminary estimates of the prevalence of selected underlying health conditions among patients with coronavirus disease 2019 d United States, february 12emarch 28 , 2020. CDC. 2020;69(13):382-386.

6. Guan W, Ni Z, Hu Y, Liang W, Ou C, He J. Clinical characteristics of coronavirus disease 2019 in China. N Engl J Med. 2020;382:17081720 .

7. Huang C, Wang Y, Li X, Ren L, et al. Clinical features of patients infected with 2019 novel coronavirus in Wuhan, China. Lancet. 2020;395(10223):497e506.

8. Wang $\mathrm{D}, \mathrm{Hu} \mathrm{B}, \mathrm{Hu} \mathrm{C}$, Zhu F, Liu X, Zhang J, et al. Clinical characteristics of 138 hospitalized patients with 2019 novel coronavirus infected pneumonia in wuhan, China. J Am Med Assoc. 2020;323(11):1061e9.

9. Yang JK, Lin SS, Ji XJ, Guo LM. Binding of SARS coronavirus to its receptor damages islets and causes acute diabetes. Acta Diabetol. 2010;47(3):193e9.

10. Akhtar H, Bishwajit B, Nayla. CM. COVID-19 and diabetes: Knowledge in progress. Diabetes Res Clin Pract 2020; 162: 108-142.

11. Wong SF, Chow KM, de Swiet M. Severe acute respiratory syndrome and pregnancy. BJOG. 2003;110:641-2.

12. Yu N, Li W, Kang Q, Xiong Z, Wiang S, Lin X. Clinical features and obstetric and neonatal outcomes of pregnant patients with COVID-19 in Wuhan, China: a retrospective, single-centre, descriptive study. Lancet Infect Dis. 2020;20:559-64.

13. Iqbal S, Overcash R, Mokhtari N, Saeed H, Gold S, Auguste T, et al. An Uncomplicated Delivery in a Patient with Covid-19 in the United States. N Engl J Med. 2020;382:16-34.

14. Zhou F, Yu T, Du R, Fan G, Liu Y, Liu Z, et al. Clinical course and risk factors for mortality of adult inpatients with COVID-19 in Wuhan, China: a retrospective cohort study. Lancet. 2020;395:1054 62.

15. Chen H, Guo J, Wang C, Luo F, Yu X, Zhang W. Clinical 
characteristics and intrauterine vertical transmission potential of COVID-19 infection in nine pregnant women: a retrospective review of medical records. Lancet. 2020;395:809-15.

16.Singh AK, Gupta R, Gosh A, Misra A. Diabetes in COVID-19: Prevalence, pathophysiolo gy, prognosis and practical considerations. Diabetes \& Metabolic Syndrome: Clin Res Rev. 2020;14:303-310.

17. Wang X, Zhou Z, Zhang J, Zhu F, Tang Y, Shen X. A Case of 2019 Novel Coronavirus in a Pregnant Woman with Preterm Delivery. Clin Infect Dis. 2020;71(15):844-6. 